\title{
Evolution of carbonic anhydrases in fungi
}

\author{
Skander Elleuche $\cdot$ Stefanie Pöggeler
}

Received: 22 January 2009 / Revised: 26 February 2009 / Accepted: 28 February 2009 / Published online: 19 March 2009

(C) The Author(s) 2009. This article is published with open access at Springerlink.com

\begin{abstract}
The ubiquitous metalloenzyme carbonic anhydrase (CA) catalyzes the interconversion of carbon dioxide and bicarbonate. This enzyme has been investigated in mammals, plants, algae, bacteria, archaea and fungi. Based on distinct structural characteristics, CAs can be assigned to five independently evolved classes $(\alpha, \beta, \gamma, \delta$ and $\zeta) . \beta$-CAs can be further subdivided into plant-type and cab-type subclasses. The recent characterization of CAs in fungi led us to initiate a systematic search for these enzymes in filamentous ascomycetes. The genomes of basidiomycetes and hemiascomycetous yeasts contain only $\beta$-CAs, while the filamentous ascomycetes also possess genes encoding $\alpha$-class CAs. Here, we present a phylogenetic analysis of 97 fungal CA sequences that addresses the diversification of fungal CAs. During evolution various gene duplication and gene loss events seem to be the cause for the multiplicity of CAs in filamentous ascomycetes. Our data revealed that during the evolution of filamentous ascomycetes, a gene encoding the plant-type $\beta$-CA was duplicated, resulting in two closely related isoforms, one with and one without an $\mathrm{N}$-terminal mitochondrial target sequence (MTS). The
\end{abstract}

Communicated by S. Hohmann.

Electronic supplementary material The online version of this article (doi:10.1007/s00294-009-0238-x) contains supplementary material, which is available to authorized users.

S. Elleuche $\cdot$ S. Pöggeler $(\bowtie)$

Department of Genetics of Eukaryotic Microorganisms, Institute of Microbiology and Genetics,

Georg-August University Göttingen,

Grisebachstr. 8, 37077 Göttingen, Germany

e-mail: spoegge@gwdg.de

S. Elleuche

Faculty of Biology and Biotechnology,

Ruhr-University of Bochum, Bochum, Germany acquisition of the MTS most likely took place after the gene duplication event and after the evolutionary separation of the fungal orders Sordariales and Eurotiales.

Keywords Carbon dioxide $\cdot$ Bicarbonate $\cdot$ Filamentous ascomycetes $\cdot$ Gene duplication $\cdot$ Mitochondrial localization
Abbreviations
$\mathrm{CA}$ Carbonic anhydrase
$\mathrm{CO}_{2} \quad$ Carbon dioxide
$\mathrm{HCO}_{3}{ }^{-}$Bicarbonate
MTS Mitochondrial target sequence
CAS Carbonic anhydrase of Sordaria
RC Reliability class
CARP Carbonic anhydrase related protein

\section{Introduction}

Carbonic anhydrases (CA) are zinc-containing metalloenzymes that catalyze the reversible hydration of carbon dioxide $\left(\mathrm{CO}_{2}\right)$ to bicarbonate $\left(\mathrm{HCO}_{3}{ }^{-}\right)$. The chemical reaction is summarized in the following equation: $\mathrm{CO}_{2}+\mathrm{H}_{2} \mathrm{O} \leftrightarrow$ $\mathrm{HCO}_{3}{ }^{-}+\mathrm{H}^{+}$. Since $\mathrm{HCO}_{3}{ }^{-}$is a substrate for many biological processes and $\mathrm{CO}_{2}$ is produced as a waste product in respiration by all organisms, the spontaneously balanced interconversion of both molecules must be regulated. CAs are distributed among all domains of life and are currently divided into five different, evolutionary unrelated classes $(\alpha, \beta, \gamma, \delta$ and $\zeta)$, which independently evolved similar catalytic mechanisms. Animal CAs belong exclusively to the group of $\alpha$-CAs. In mammals, 16 different tissue- and organ-specific $\alpha$-CA isoforms or CA-related proteins (CARP) have been described (Supuran 2008). These are 
localized to different subcellular compartments, including five cytoplasmic CAs, two mitochondrially targeted, one secreted and five membrane-associated isoforms (Kivelä et al. 2005; Supuran 2008). Phylogenetic analyses revealed that all human $\alpha$-CA isoforms clearly originated from a common ancestor (Hewett-Emmett and Tashian 1996; Mori et al. 1999). In addition to animals, $\alpha$-class CAs have also been identified in prokaryotes, plants, algae and fungi (Moroney et al. 2001; Tripp et al. 2001; Bahn and Mühlschlegel 2006). $\beta$-CAs are present in algae, plants, fungi and bacteria, whereas $\gamma$-CAs with strikingly different sequence features were predominantly found in archaea and some bacteria (Alber and Ferry 1994; Hewett-Emmett and Tashian 1996; Smith and Ferry 2000; Moroney et al. 2001). The $\beta$-class can be further subdivided into two main subclasses, the plant-type and the cab-type class, named after the $\beta$-CA CAB from the archaeon Methanobacterium thermoautotrophicum (Smith and Ferry 1999; Kimber and Pai 2000). While the active-site residues Gln151, Phe179 and Tyr205 are conserved in plant-type $\beta$-CAs (numbering according to the Pisum sativum $\mathrm{CA}$ ), they are variable in the cab-type $\beta$-CAs. A third sub-class of $\beta$-CAs, primarily designated as $\varepsilon$-class, was found to be present in the chemolithoautotrophic bacterium Halothiobacillus neapolitanus (So et al. 2004; Sawaya et al. 2006). The $\delta$ - and $\zeta$-classes of CAs are so far restricted to marine diatoms (Lane et al. 2005; Park et al. 2007).

The molecular structures of the various classes of CAs are conspicuously different, but the metal coordinating active sites are remarkably similar at the structural level (Liljas and Laurberg 2000). A principle difference between CAs is the composition of their subunits. Whereas $\alpha$-CAs are composed of one monomer, $\beta$-class CAs form oligomers with two to six monomers and the prototype $\gamma$-CA from Methanosarcina thermophila is a homotrimer (Supuran 2008). The lack of significant sequence similarities between different classes of CAs makes them excellent examples of convergent evolution of catalytic function.

Only recently have fungal CAs been analysed in detail. The first fungal CA was discovered in the yeast Saccharomyces cerevisiae and belongs to the $\beta$-class. The $S$. cerevisiae $\beta$-CA was shown to be essential under ambient air conditions and transcriptionally regulated by the concentration of inorganic carbon (Götz et al. 1999; Amoroso et al. 2005). Furthermore, one $\beta$-CA-gene was found in the pathogenic yeast Candida albicans and two in the pathogenic basidiomycete Cryptococcus neoformans (Bahn et al. 2005; Klengel et al. 2005). Deletion of the C. albicans nce103 gene resulted in a lethal phenotype under low $\mathrm{CO}_{2}$-conditions, whereas in $C$. neoformans only can2, encoding the major CA, was shown to be essential under ambient air conditions. Heterologous expression of can 2 from C. neoformans completely restored the high $\mathrm{CO}_{2}$-requiring pheno- types of S. cerevisiae and E. coli (Bahn et al. 2005; Mogensen et al. 2006). In the filamentous ascomycete Sordaria macrospora, three active $\beta$-CA isoforms (CAS1, CAS2 and CAS3, carbonic anhydrase of Sordaria) have been characterized. CAS1 and CAS2 belong to plant-type $\beta$-CAs, whereas CAS3 can be classified as a cab-type $\beta$-CA. Localization studies revealed that CAS2 is translocated into mitochondria, while CAS1 and CAS3 are cytoplasmic enzymes. A genetic analysis of knock-out strains $\Delta$ cas $1, \Delta$ cas 2 and $\Delta$ cas 3 , demonstrated that CAS1 and CAS2 are involved in fruiting body and ascospore formation (S. Elleuche and S. Pöggeler, unpublished data).

The rapidly increasing number of published fungal genome sequences makes it possible to reconstruct the evolutionary history of fungal CAs. In this study, we have examined the evolution of the fungal CAs. Three isoforms (CAS1-, CAS2- and CAS3-homologues) of $\beta$-class CAs and at least one $\alpha$-class CA are commonly found in the genomes of filamentous ascomycetes, whereas most hemiascomycetous yeasts contain only one $\beta$-class CA. In filamentous ascomycetes, CAS1 and CAS2 are two homologues of plant-type $\beta$-CAs that are closely related and might be the result of a recent gene duplication event. Interestingly, the cas 1 -homologues encoded by members of the class Eurotiomycetes exhibit a mitochondrial target sequence (MTS), whereas an analogous target peptide is encoded at the 5'-end of cas2-homologues in Sordariomycetes. Evolutionary relations of fungal CAs are discussed.

\section{Materials and methods}

\section{Sequence analysis}

Fungal genomic sequences used for this study are available at: Fungal Genome Initiative (Broad Institute: http://www. broad.mit.edu/annotation/fgi/), The DOE Joint Genome Institute (JGI:http://www.jgi.doe.gov/), Génolevures (http:/ /cbi.labri.fr/Genolevures/) and the Institut de Génétique et Microbiologie-Université de Paris-Sud XI/CNRS (Espagne et al. 2008). All downloads were performed before 1 December 2008.

Sequences of $\beta$-CAs from Saccharomyces cerevisiae (Nce103p-NP_014362.1), Candida albicans (NCE103Q5AJ71) and Cryptococcus neoformans (CAN1-AAZ 30050.1; CAN2-AAZ30051.1) were used as described previously (Götz et al. 1999; Bahn et al. 2005; Klengel et al. 2005). To identify fungal $\alpha$ - and $\beta$-CAs, we performed blastp, tblastn (Altschul et al. 1997) and key word searches. Annotations of several mitochondrial CAs were found to be incorrect, because MTS were not detectable under the given annotated genes or accession numbers. As long as MTS could be found within the accordant open 
reading frames or in the sequence preceding the gene, we referred to in Supplementary Figure 3 and in "Results".

Phylogenetic analysis

Multiple protein sequence alignments were performed using the clustalX program (Thompson et al. 2002). Phylogenetic analysis was made with programs from package PHYLIP version 3.6 (http://evolution.genetics.washington.edu/phylip.html). PROTPARS was used to construct phylogenetic trees, evaluating statistical significance by bootstrap analysis with 1,000 iterations of bootstrap samplings and reconstruction of trees by PROTPARS. A majority rule consensus tree was subsequently generated with the program CONSENSE, viewed using the program TreeView (Win 32) 1.6.6 (Page 1996) and saved for graphical representation using Adobe Illustrator. The phylogenetic tree was generated based on an alignment that starts with Leu $^{41}$ and ends with Gly ${ }^{105}$ of the CAS1 protein from S. macrospora (FM878639). For the phylogenetic analysis of the catalytic center of CAs, we made a modification concerning intron splicing of an annotated casl-homologue from Podospora anserina (XP_001905915.1) Our annotation increased the sequence identity to related CAs from S. macrospora or N. crassa and produced a putative CA protein.

MTS and secondary structure predictions

The programs MitoProtII-v1.101 and TargetP 1.1 were used to predict putative N-terminal MTS with their corresponding cleavage sites and to measure the probability of import into mitochondria (Claros and Vincens 1996; Emanuelsson et al. 2007). Prediction of the secondary structures of CAs from $S$. macrospora was done using the PSIPRED V2.6 software from the PSIPRED Protein Structure Prediction Server (McGuffin et al. 2000).

\section{Results}

Phylogenetic relationships of fungal carbonic anhydrases

Recent large-scale genome sequencing projects have led to the deposition of a huge number of fungal genome sequences in open-access databases. Previously, using heterologous primers based on the nucleotide sequences of Neurospora crassa CA genes, we identified three $\beta$-CA open reading frames and one $\alpha$-CA of the filamentous ascomycete $S$. macrospora, whose genome has not yet been sequenced. The according genes were designated casl, cas 2 , cas 3 and cas4 (Fig. 1). Using amino acid sequences from CAS1, CAS2 and CAS3 for blastp and tblastn
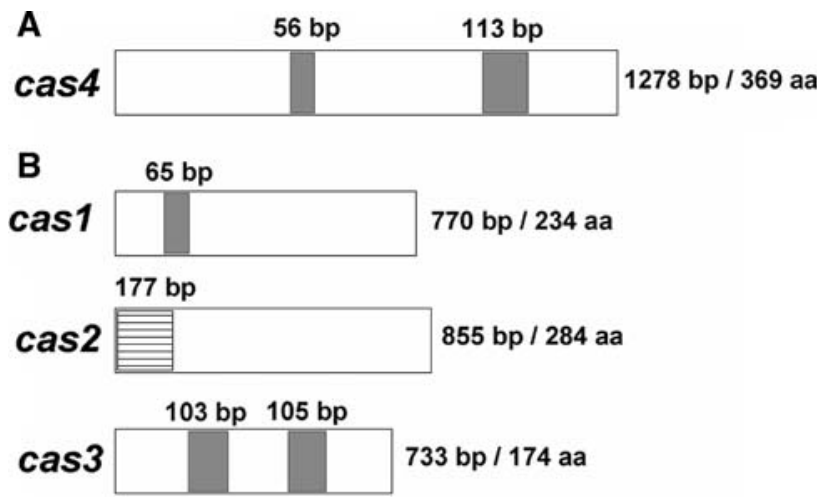

Fig. 1 Schematic representation of the four cas-genes from $S$. macrospora. a $\alpha$-CAs; $\mathbf{b} \beta$-CAs. Sizes of genes, introns (grey boxes) and of the encoded proteins are given. The mitochondrial target sequence (MTS) encoded at the $\mathrm{N}$-terminal part of cas 2 is indicated as dashed box

searches, we identified 82 fungal $\beta$-CA genes from diverse ascomycetes and basidiomycetes (Table 1). A previously identified $\alpha$-CA from Aspergillus oryzae was used to perform a systematic search on the presence of $\alpha$-CAs in fungi and produced 13 putative $\alpha$-CA genes from filamentous ascomycetes (Bahn and Mühlschlegel 2006). The $\alpha$-CA of S. macrospora has been identified by means of PCR with heterologous primers designed on the sequence of a $N$. crassa $\alpha$-CA gene.

To understand the evolutionary relationship of CAs in the fungal kingdom, the catalytic center of identified CA sequences was used to perform a maximum parsimony analysis (Fig. 2; Supplementary Figure 1). No changes in the three essential key amino acid residues are detectable in all identified fungal CAs, which have been used for the generation of the phylogenetic tree. The $\beta$-CAs are distributed over the fungal phylum, whereas $\alpha$-CAs seem to be restricted to filamentous ascomycetes and form a single clade (Fig. 2). This clade contains $\alpha$-CAs of members of the fungal orders Eurotiales, Hypocreales, Onygenales, Pleosporales and Sordariales as well as an $\alpha$-CA from Magnaporthe grisea which belongs to the class of Sordariomycetes but is not assigned to a specific order (Table 1). The clade of $\alpha$-CAs is clearly separated from the fungal $\beta$-CA sequences. Only one $\alpha$-CA gene was identified in most of the genomes analysed. Two putative $\alpha$-CA genes were identified in A. terreus and in Fusarium graminearum, whereas no $\alpha$-CA seems to be present in the Aspergillus species A. nidulans and A. fumigatus.

The three $S$. macrospora $\beta$-CAs (CAS1, CAS2, CAS3) show an obvious separation into three different clades (Fig. 2). Similar to $S$. macrospora, three CAS-homologues were identified in the following fungal orders of filamentous ascomycetes: Eurotiales (A. fumigatus, Neosartorya fischeri), Onygenales (Paracoccidioides brasiliensis), 
Table $1 \alpha$-and $\beta$-class carbonic anhydrases encoded by the nuclear genomes of ascomycetes and basidiomycetes

\begin{tabular}{|c|c|c|c|c|c|}
\hline Order & Species & Abbreviation & Accession numbers or gene numbers ${ }^{\mathrm{a}}$ & Clade in Fig. 2 & MTS \\
\hline \multicolumn{6}{|l|}{ Ascomycetes } \\
\hline \multirow[t]{17}{*}{ Eurotiales } & \multirow[t]{2}{*}{ Aspergillus clavatus } & Acl_1 & XP_001271988 & $\beta$-CAS1 & + \\
\hline & & Acl_2 & XP_001273459.1 & $\beta$-CAS2 & - \\
\hline & Aspergillus flavus & Afl_1 & EED55452 & $\beta$-CAS 1 & + \\
\hline & \multirow[t]{3}{*}{ Aspergillus fumigatus } & Afu_1 & XP_751704.1 & $\beta$-CAS1 & + \\
\hline & & Afu_2 & XP_001481412.1 & $\beta$-CAS2 & - \\
\hline & & Afu_3 & XP_751882.1 & $\beta$-CAS3 & - \\
\hline & \multirow[t]{2}{*}{ Aspergillus nidulans } & And_1 & XP_663215.1 $1^{\mathrm{b}}$ & $\beta$-CAS 1 & $(?)^{\mathrm{c}}$ \\
\hline & & And_2 & Q5BCC5 & $\beta$-CAS2 & - \\
\hline & \multirow[t]{2}{*}{ Aspergillus oryzae } & Aor_1 & XP_001820193 ${ }^{\mathrm{b}}$ & $\beta$-CAS1 & + \\
\hline & & Aor_4 & XP_001827551.1 & $\alpha$-CAS4 & - \\
\hline & \multirow[t]{4}{*}{ Aspergillus terreus } & Ate_1 & XP_001213134 & $\beta$-CAS1 & + \\
\hline & & Ate_3 & XP_001209372.1 & $\beta$-CAS3 & - \\
\hline & & Ate_4a & XP_001210252.1 & $\alpha$-CAS4 & - \\
\hline & & Ate_4b & XP_001215283.1 & $\alpha$-CAS4 & - \\
\hline & \multirow[t]{3}{*}{ Neosartorya fischeri } & Nfi_1 & XP_001266926 & $\beta$-CAS1 & + \\
\hline & & Nfi_2 & XP_001262181.1 & $\beta$-CAS2 & - \\
\hline & & Nfi_3 & XP_001267068.1 & $\beta$-CAS3 & - \\
\hline \multirow[t]{3}{*}{ Helotiales } & \multirow[t]{2}{*}{ Botryotinia fuckeliana } & Bfu_1 & XP_001555448.1 & $\beta$-CAS1 & - \\
\hline & & Bfu_3 & XP_001561102.1 & $\beta$-CAS3 & - \\
\hline & Sclerotinia sclerotiorum & Ssc_1 & XP_001598060.1 & $\beta$-CAS1 & - \\
\hline \multirow[t]{27}{*}{ Hypocreales } & \multirow[t]{5}{*}{ Fusarium graminearum } & Fgr_2 & XP_384734.1 ${ }^{\mathrm{b}}$ & $\beta$-CAS2 & + \\
\hline & & Fgr_3a & XP_383146.1 & $\beta$-CAS3 & - \\
\hline & & Fgr_3b & XP_390629 & $\beta$-CAS3 & - \\
\hline & & Fgr_4a & XP_391271.1 & $\alpha$-CAS4 & - \\
\hline & & Fgr_4b & XP_384779.1 & $\alpha$-CAS4 & - \\
\hline & \multirow[t]{3}{*}{ Fusarium oxysporum } & Fox_2 & supercont $2.20^{\mathrm{b}}$ & $\beta$-CAS2 & + \\
\hline & & Fox_3a & FOXG_12252.2 & $\beta$-CAS3 & - \\
\hline & & Fox_3b & FOXG_13574.2 & $\beta$-CAS3 & - \\
\hline & \multirow[t]{3}{*}{ Fusarium verticillioides } & Fve_2 & supercont_3.16 ${ }^{\mathrm{b}}$ & $\beta$-CAS2 & + \\
\hline & & Fve_3a & FVEG_10874.3 & $\beta$-CAS3 & - \\
\hline & & Fve_3b & FVEG_01549.3 & $\beta$-CAS3 & - \\
\hline & \multirow[t]{6}{*}{ Trichoderma atroviride } & Tat_2 & Triat1/scaffold_4 ${ }^{\mathrm{b}}$ & $\beta$-CAS2 & + \\
\hline & & Tat_3a & Triat1/scaffold_13 & $\beta$-CAS3 & - \\
\hline & & Tat_3b & Triat1/scaffold_5 & $\beta$-CAS3 & - \\
\hline & & Tat_3c & Triat1/scaffold_18 & $\beta$-CAS3 & - \\
\hline & & Tat_3d & Triat1/scaffold_14 & $\beta$-CAS3 & - \\
\hline & & Tat_4 & Triat1/scaffold_17 & $\alpha$-CAS4 & - \\
\hline & \multirow[t]{5}{*}{ Trichoderma reesei } & Tre_2 & Trire2/scaffold_25 ${ }^{\mathrm{b}}$ & $\beta$-CAS2 & + \\
\hline & & Tre_3a & Trire2/scaffold_30 & $\beta$-CAS3 & - \\
\hline & & Tre_3b & Trire2/scaffold_5 & $\beta$-CAS3 & - \\
\hline & & Tre_3c & Trire2/scaffold_3 & $\beta$-CAS3 & - \\
\hline & & Tre_4 & Trire2/scaffold_32 & $\alpha$-CAS4 & - \\
\hline & \multirow[t]{5}{*}{ Trichoderma virens } & Tvi_2 & Trive1/scaffold_4 ${ }^{\mathrm{b}}$ & $\beta$-CAS2 & + \\
\hline & & Tvi_3a & Trive1/scaffold_15 & $\beta$-CAS3 & - \\
\hline & & Tvi_3b & Trive1/scaffold_12 & $\beta$-CAS3 & - \\
\hline & & Tvi_3c & Trive1/scaffold_13 & $\beta$-CAS3 & - \\
\hline & & Tvi_4 & Trive1/scaffold_8 & $\alpha$-CAS4 & - \\
\hline
\end{tabular}


Table 1 continued

\begin{tabular}{|c|c|c|c|c|c|}
\hline Order & Species & Abbreviation & Accession numbers or gene numbers ${ }^{\mathrm{a}}$ & Clade in Fig. 2 & MTS \\
\hline \multirow[t]{6}{*}{ Onygenales } & \multirow[t]{2}{*}{ Coccidioides immitis } & Cim_1 & XP_001247766.1 & $\beta$-CAS1 & - \\
\hline & & Cim_3 & XP_001241380.1 & $\beta$-CAS3 & - \\
\hline & \multirow[t]{4}{*}{ Paracoccidioides brasiliensis } & Pbr_1 & PADG_01697.1 & $\beta$-CAS1 & - \\
\hline & & Pbr_2 & supercont $1.12^{\mathrm{b}}$ & $\beta$-CAS2 & + \\
\hline & & Pbr_3 & PADG_00315.1 & $\beta$-CAS3 & - \\
\hline & & Pbr_4 & ACA28690.1 & $\alpha$-CAS4 & - \\
\hline \multirow[t]{4}{*}{ Pleosporales } & \multirow[t]{4}{*}{ Phaeosphaeria nodorum } & Pno_1 & XP_001797035.1 & $\beta$-CAS1 & - \\
\hline & & Pno_2 & XP_001802070.1 & $\beta$-CAS2 & + \\
\hline & & Pno_3 & XP_001805507.1 & $\beta$-CAS3 & - \\
\hline & & Pno_4 & XP_001790777.1 & $\alpha$-CAS4 & - \\
\hline \multirow[t]{16}{*}{ Sordariales } & \multirow[t]{4}{*}{ Chaetomium globosum } & Cgl_1 & XP_001226871.1 & $\beta$-CAS1 & - \\
\hline & & Cgl_2 & XP_001227705 & $\beta$-CAS2 & + \\
\hline & & Cgl_3 & XP_001225170.1 & $\beta$-CAS3 & - \\
\hline & & Cgl_4 & XP_001227267.1 & $\alpha$-CAS4 & - \\
\hline & \multirow[t]{4}{*}{ Neurospora crassa } & Ncr_1 & XP_960227 & $\beta$-CAS1 & - \\
\hline & & Ncr_2 & XP_959676 ${ }^{\mathrm{b}}$ & $\beta$-CAS2 & + \\
\hline & & Ncr_3 & XP_961715 & $\beta$-CAS3 & - \\
\hline & & Ncr_4 & XP_960214 & $\alpha$-CAS4 & - \\
\hline & \multirow[t]{4}{*}{ Podospora anserina } & Pan_1 & XP_001905915.1 & $\beta$-CAS 1 & - \\
\hline & & Pan_2 & XP_001905568.1 & $\beta$-CAS2 & + \\
\hline & & Pan_3 & XP_001911575.1 & $\beta$-CAS3 & - \\
\hline & & Pan_4 & XP_001906308.1 & $\alpha$-CAS4 & - \\
\hline & \multirow[t]{4}{*}{ Sordaria macrospora } & Sma_1 & FM878639 & $\beta$-CAS1 & - \\
\hline & & Sma_2 & FM878640 & $\beta$-CAS2 & + \\
\hline & & Sma_3 & FM878641 & $\beta$-CAS3 & - \\
\hline & & Sma_4 & FN178637 & $\alpha$-CAS4 & - \\
\hline \multirow[t]{4}{*}{ "Not assigned" } & \multirow[t]{4}{*}{ Magnaporthe grisea } & Mgr_1 & XP_362166.2 & $\beta$-CAS1 & - \\
\hline & & Mgr_2 & XP_366523 ${ }^{\mathrm{b}}$ & $\beta$-CAS2 & + \\
\hline & & Mgr_3 & XP_364389.1 & $\beta$-CAS3 & - \\
\hline & & Mgr_4 & XP_363766.1 & $\alpha$-CAS4 & - \\
\hline \multicolumn{6}{|l|}{ Hemiascomycetous yeasts } \\
\hline \multirow[t]{13}{*}{ Saccharomycetales } & Ashbya gossypii & Ago & NP_983870.1 & $\beta$-CA Yeasts & - \\
\hline & \multirow[t]{2}{*}{ Candida albicans } & $\mathrm{Cal}$ & Q5AJ71 & $\beta$-CA Yeasts & - \\
\hline & & Cal_3 & XP_715817 & $\beta$-CAS3 & - \\
\hline & Candida glabrata & $\mathrm{Cgl}$ & XP_446428.1 & $\beta$-CA Yeasts & - \\
\hline & Debaryomyces hansenii & Dha & XP_456870.1 & $\beta$-CA Yeasts & - \\
\hline & Kluyveromyces lactis & Kla & XP_455263.1 & $\beta$-CA Yeasts & - \\
\hline & Lodderomyces elongisporus & Lel & XP_001527257.1 & $\beta$-CA Yeasts & - \\
\hline & Pichia guilliermondii & Pgu & EDK37806.2 & $\beta$-CA Yeasts & - \\
\hline & \multirow[t]{2}{*}{ Pichia stipitis } & Pst & XP_001386459.2 & $\beta$-CA Yeasts & - \\
\hline & & Pst_3 & XP_001383682.1 & $\beta$-CAS3 & - \\
\hline & Saccharomyces cerevisiae & Sce & NP_014362.1 & $\beta$-CA Yeasts & - \\
\hline & Vanderwaltozyma polyspora & Vpo & XP_001644582.1 & $\beta$-CA Yeasts & - \\
\hline & Yarrowia lipolytica & Yli & XP_505708.1 & $\beta$-CA Yeasts & - \\
\hline Schizosaccharomycetales & Schizosaccharomyces pombe & Spo & NP_596512.1 & $\beta$-CA Yeasts & - \\
\hline \multicolumn{6}{|c|}{ Basidiomycetes } \\
\hline \multirow[t]{2}{*}{ Agaricales } & Coprinopsis cinerea & Cci & XP_001833059.1 & $\beta$-CA Basidiomycetes & - \\
\hline & Laccaria bicolor & Lbi & XP_001882562.1 & $\beta$-CA Basidiomycetes & - \\
\hline
\end{tabular}


Table 1 continued

\begin{tabular}{|c|c|c|c|c|c|}
\hline Order & Species & Abbreviation & Accession numbers or gene numbers ${ }^{\mathrm{a}}$ & Clade in Fig. 2 & MTS \\
\hline Malasseziales & Malassezia globosa & $\mathrm{Mgl}$ & XP_001730815.1 & $\beta$-CA Basidiomycetes & - \\
\hline \multirow[t]{2}{*}{ Tremellales } & Cryptococcus neoformans & Cne_1 & AAZ30050.1 & $\beta$-CA Basidiomycetes & - \\
\hline & & Cne_2 & AAZ30051.1 & $\beta$-CA Basidiomycetes & - \\
\hline Ustilaginales & Ustilago maydis & Uma & XP_756348.1 & $\beta$-CA Basidiomycetes & - \\
\hline
\end{tabular}

${ }^{a}$ Abbreviations and accession numbers or numbers of hypothetical proteins from genome projects or scaffolds are given

b Annotated ORFs were modified for MTS identification

c A putative MTS in the $\beta$-CAS1-homologue from A. nidulans was not detected

Pleosporales (Phaeosphaeria nodorum), Sordariales (Chaetomium globosum, N. crassa, Podospora anserina) and Magnaporthe grisea. While homologues of CAS1 and CAS2 belong to the sub-group of plant-type CAs and are closely related to each other, cab-type CAS3-homologues are quite distantly related to CAS1 and CAS2. This can also be seen by comparing the predicted secondary structure of the $S$. macrospora $\beta$-CAs (Supplementary Figure 3). CAS1 and CAS2 homologues show a fairly high degree of sequence identity in all species investigated (between 30 and 50\% sequence identity over most of the protein length and up to $72.3 \%$ at the catalytic region). Comparison of cab-type CAS3 homologues with either CAS1- or CAS2homologues resulted in only $25-30 \%$ sequence identity within the catalytic region. Interestingly, members of the Hypocreales exhibit multiple closely related cas 3 homologues encoding cab-type $\beta$-CAs $(F$. graminearum, $F$. oxysporum, $F$. verticillioides, Trichoderma atroviride, $T$. reesei and $T$. virens). However, only one cas2-homologue is encoded by members of the order Hypocreales and a homologue of casl is completely missing (Table 1; Fig. 2).

In contrast to the multiple CA-genes of filamentous ascomycetes, most hemiascomycetous yeasts and basidiomycetes encode only a single CA belonging to the planttype sub-group. Additionally, putative cab-type $\beta$-CAs have been identified in the genomes of the hemiascomycetous yeasts Candida albicans (XP_715817) and Pichia stipitis (XP_001383682.1).

Within the basidiomycetes, $C$. neoformans encodes two $\beta$-CAs, whereas a single $\beta$-CA gene was identified in Coprinopsis cinerea, Laccaria bicolor, Malassezia globosa and Ustilago maydis.

\section{Mitochondrial localization of CAS-homologues}

In S. macrospora, mitochondrial localization of CAS2 is required for ascospore germination. A $\Delta$ cas2-deletion strain could not be complemented with a cytoplasmic CAS2-variant (S. Elleuche and S. Pöggeler, unpublished data). With this in mind, we tried to identify putative MTS in the CAs of filamentous fungi, but most of the fungal CA-genes deposited into databases are annotated without MTS. By changing the annotation of the first exon of casl- and cas2homologues we identified putative MTS in several deduced amino acid sequences. In contrast, no MTS were found in CAS3 homologues and fungal $\alpha$-class CAs.

The gene $N$. crassa NCU08133.3 has been annotated with a CTG-triplet as start-codon (XP_959676), which would lead to the loss of the MTS and a non-functional CAvariant of the homologous CAS2-protein in the closely

Fig. 2 Unrooted phylogenetic tree of $\alpha$ - and $\beta$-class CAs from ascomycetes and basidiomycetes. The phylogenetic tree was made with programs from the program package PHYLIP, based on a clustalX alignment of the catalytic region from CA proteins. Abbreviations do not specify characterized genes, but are chosen to simplify the presented data. Accession numbers or numbers of hypothetical proteins or scaffolds from genome projects are given in Table 1. Ashbya gossypii (Ago), Aspergillus clavatus (Acl_1; Acl_2), Aspergillus flavus (Afl_1), Aspergillus fumigatus (Afu_1; Afu_2; Afu_3), Aspergillus nidulans (And_1; And_2), Aspergillus oryzae (Aor_1; Aor_4), Aspergillus terreus (Ate_1; Ate_3; Ate_4a; Ate_4b), Botryotinia fuckeliana (Bfu_1; Bfu_3), Candida albicans (Cal; Cal_3), Candida glabrata (Cgl), Chaetomium globosum (Cgl_1; Cgl_2; Cgl_3; Cgl_4), Coccidioides immitis (Cim_1; Cim_3), Coprinopsis cinerea (Cci), Cryptococcus neoformans (Cne1; Cne2), Debaryomyces hansenii (Dha), Fusarium graminearum (Fgr_2; Fgr_3; Fgr_4; Fgr_4a; Fgr_4b), Fusarium oxysporum (Fox_2; Fox_3; Fox_4), Fusarium verticillioides (Fve_2; Fve_3; Fve_4), Kluyveromyces lactis (Kla), Laccaria bicolor (Lbi), Lodderomyces elongisporus (Lel), Magnaporthe grisea (Mgr_1; Mgr_2; Mgr_3; Mgr_4), Malassezia globosa (Mgl), Neosartorya fischeri (Nfi_1; Nfi_2; Nfi_3), Neurospora crassa (Ncr_1; Ncr_2; Ncr_3; Ncr_4), Paracoccidioides brasiliensis (Pbr_1; Pbr_2; Pbr_3; Pbr_4), Phaeosphaeria nodorum (Pno_1; Pno_2; Pno_3; Pno_4), Pichia guilliermondii (Pgu), Pichia stipitis (Pst; Pst_3), Podospora anserina (Pan_1; Pan_2; Pan_3; Pan_4), Saccharomyces cerevisiae (Sce), Schizosaccharomyces pombe (Spo), Sclerotinia sclerotiorum (Ssc_1), Sordaria macrospora (Sma_1; Sma_2; Sma_3; Sma_4), Trichoderma atroviride (Tat_2; Tat_3a; Tat_3b; Tat_3c; Tat_3d; Tat_4), Trichoderma reesei (Tre_2; Tre_3a; Tre_3b; Tre_3c; Tre_4), Trichoderma virens (Tvi_2; Tvi_3a; Tvi_3b; Tvi_3c; Tvi_3d; Tvi_4), Ustilago maydis (Uma), Vanderwaltozyma polyspora (Vpo), Yarrowia lipolytica (Yli). All fungal CAs are clearly separated to six different branches. The $\beta$-class CAs from filamentous ascomycetes all belong to three different groups, indicated as CAS1-3-homologues (CAS carbonic anhydrase of Sordaria, indicated as Sma_1,Sma_2,Sma_3 and Sma_4 in the phylogenetic tree). All $\beta$-CAs that exhibit a mitochondrial target sequence (CAS1 and CAS2 homologues) are in white and highlighted in black. The numbers at the nodes indicate the percentage of bootstrap support 


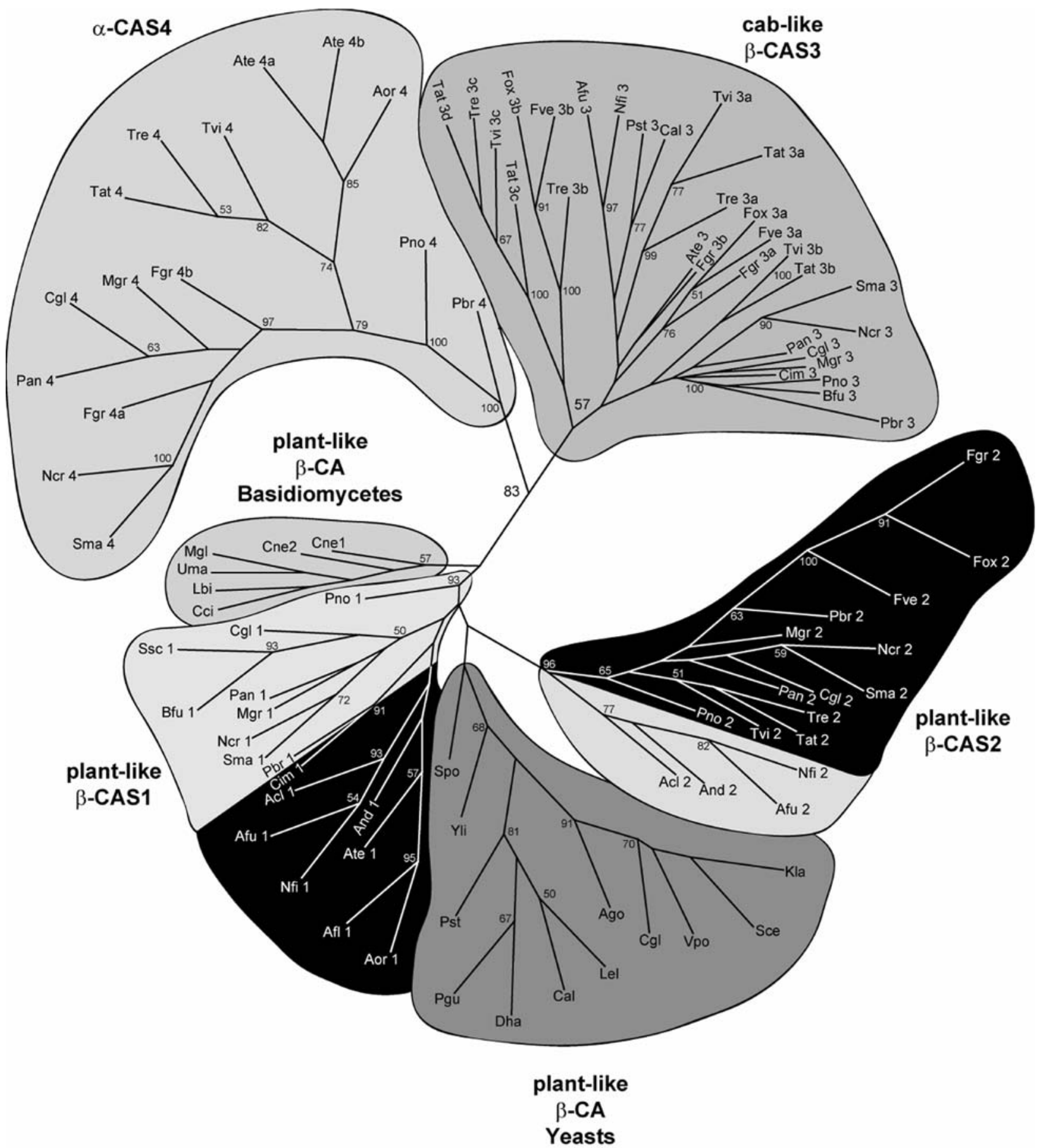

related species $S$. macrospora (Fig. 3a). An ATG-codon is located 102-bp upstream of the CTG-codon in $N$. crassa and might be the functional start codon in this fungus by comparison with the homologous ATG-start codon in the cas2 gene of S. macrospora. Programs MitoProtII and TargetP predicted the MTS of $S$. macrospora and N. crassa to contain 59-aa residues (Fig. 4) and share a high degree of identity ( $74.6 \%$ identity in 59 -aa overlap). In contrast, the 59-aa MTS of Podospora anserina and the 58-aa MTS of the more distantly related Phaeosphaeria nodorum exhibit less similarity (32.4\% in 34 aa and 53.8\% in 13 aa overlap identity to $S$. macrospora). Similar to $N$. crassa, no MTS was annotated for the cas2-homologue of Chaetomium globosum (XP_001227705). However, a second ATGcodon is located in the upstream region (239 bp upstream of the annotated start codon) of the annotated start-codon 
A

Neurospora crassa

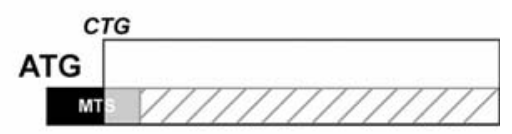

B

Aspergillus terreus

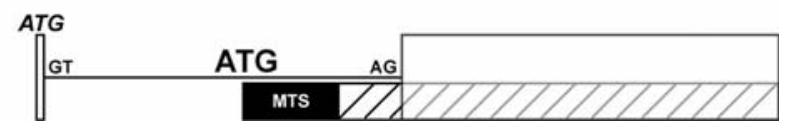

Fig. 3 Structure of the deposited and improved annotated cas 2 gene from $N$. crassa and cas 1 gene from A. terreus. a The N. crassa cas2homologue is annotated starting with a CTG-codon and with a truncated 25-aa part of the MTS. Using an ATG-start codon upstream of the CTG results in a deduced amino acid sequence with a putative $\mathrm{N}$ terminal MTS. b The deposited cas1-homologue of A. terreus is interrupted by a putative 595-bp intron, and encodes no MTS. Starting translation of the ORF at an ATG within the predicted intron results in a deduced amino acid sequence with a putative N-terminal MTS. Deposited gene-variants are indicated as white boxes. Coding sequences of MTS are indicated as black boxes preceding the newly annotated full length ORFs given as dashed boxes. The thin line indicates the predicted intron in the A. terreus cas 1 gene with conserved 5 '-donor and 3 '-acceptor sequences. The predicted start codons are indicated in bold italics (CTG in case of N. crassa and ATG in case of A. terreus). ATG in bold at the beginning of the accordant MTS indicates the newly annotated start codons

(Supplementary Figure 3). In silico analysis of this region resulted in the detection of a putative MTS, but the upstream ATG-codon is not in-frame with the annotated ORF. For this ORF, we assume that a nucleotide is missing in the genome sequence and predict that the CAS2 homologue of C. globosum possesses a MTS.

Similarly, within the $5^{\prime}$-upstream region of the annotated cas2-homologues of the order Hypocreales, an alternative ATG-start codon was identified (Supplementary Figure 3), encoded by the upstream nucleotides -213 to -211 in F. graminearum (supercont_3.2), nucleotides -204 to -202 in F. oxysporum (supercont_2.20) and -189 to -187 in case of $F$. verticillioides (supercont_3.16). This resulted in the prediction of a 44-aa MTS in F. oxysporum, a 46-aa MTS in F. verticillioides and a 71-aa MTS in F. graminearum (Fig. 4). Comparable results were obtained my modifying the annotated ORFs from three species of the genus Trichoderma. Translation starting with upstream ATG-start codons resulted in the identification of a 33-aa, 37-aa and a 13-aa MTS in T. atroviride, T. reesei and T. virens, respectively (Supplementary Figure 3).

In Coccidioides immitis (Onygenales), the casl-homologue encodes a protein that contains a putative 33-aa MTS, whereas in Paracoccidioides brasiliensis (Onygenales), the cas2-homologue encodes a protein with a putative MTS of 66 aa (supercont1.12, Fig. 4). The target peptides of both proteins are highly dissimilar not only in length, but also in their sequences (31.8\% in 22 aa overlap).

Similar to CAS1 of C. immitis, casl-homologues of members of the Eurotiales encode proteins with MTS. In $A$. oryzae (XP_001820193) and A. terreus (XP_001213134), casl genes are annotated with an intron (A. oryzae 615-bp, A. terreus 595-bp). The sequence of both introns contains an alternative ATG-start codon, and translation without the preceding intron splicing would lead to the expression of $\beta$-CA-variants with putative MTS of 39 and 61 aa, respectively (Figs. 3b, 4; Supplementary Figure 3). The A. clavatus cas 1 -homologue (XP_001271988) has also been annotated without a MTS. Here, translation from a 135-bp upstream ATG-start codon leads to the creation of a putative MTS of 25 aa. In the same manner, we annotated the casl-homologue of Neosartorya fischeri (NFIA_105170), resulting in the prediction of a MTS containing 25-aa residues (Supplementary Figure 3). The casl-homologue from A. fumigatus (XP_751704.1) is annotated with the nucleotides encoding for a putative 27-aa MTS, which is homologous and highly similar ( $84.0 \%$ identity in 25 aa overlap) to our newly annotated MTS of $N$. fischeri.

\section{Discussion}

To date, functional characterizations of fungal $\beta$-CAs have been done only on the hemiascomycetous yeasts $S$. cerevisiae, the human pathogen $C$. albicans and the basidiomycetous fungus $C$. neoformans (Götz et al. 1999; Bahn et al. 2005; Klengel et al. 2005). In this study, we performed genome wide searches of fungal genomes for genes encoding CAs to provide a detailed survey of the distribution of CAs in fungi. At least one $\beta$-CA has been identified in all fungi investigated, whereas $\alpha$-CAs have been found in filamentous ascomycetes, but not in hemiascomycetous yeasts and basidiomycetes. Previously, Bahn and Mühlschlegel (2006) identified a single putative $\alpha$-CA gene of A. oryzae, in addition to $\beta$-CAs. Using the $\alpha$-CA sequence from A. oryzae, we identified 14 additional $\alpha$-CAs within the fungal orders Eurotiales, Hypocreales, Onygenales and Sordariales. In the genomes of $F$. graminearum and $A$. terreus two $\alpha$-CAs with a high degree of amino acid identity were identified, indicating putative gene duplication events within these two species (Fig. 5). Interestingly, while at least one $\alpha$-CA gene is present in the Aspergillus species A. terreus and $A$. oryzae as well as in A. flavus and A. niger (data not shown), we were unable to identify $\alpha$-CA-genes in the genomes of A. nidulans, A. fumigatus, N. fischeri and A. clavatus. A recent comparative analysis of the genomes of A. nidulans, A. fumigatus and A. oryzae, revealed that A. oryzae has approximately $20 \%$ more genes than its congeneric species A. nidulans and A. fumigatus. Many of 
Fig. 4 Mitochondrial target sequence predictions of $\beta$-CAs from filamentous ascomycetes. CAs were depicted as white boxes with MTS indicated as grey boxes. Number of amino acid residues of the MTS is given. The probability of import into mitochondria is indicated as values derived from predictions with programs MitoProtII and TargetP. According to TargetP, the reliability was classified (RC) with $R C l$ indicating the strongest and $R C 5$ the lowest reliability of the prediction (http://www.cbs.dtu.dk/ services/TargetP-1.1/ output.php)

Sordaria macrospora
Neurospora crassa
Podospora anserina
Magnaporthe grisea
Phaeosphaeria nodorum
Fusarium graminearum
Fusarium verticillioides
Fusarium oxysporum
Trichoderma atroviride
Trichoderma reesei
Trichoderma virens
Paracoccidioides brasiliensis
Aspergillus niger
Aspeosartorya fischeri
Aspergillus flavus
Aspergillus oryzae
Aspergillillus terreus

\begin{tabular}{|c|c|c|c|}
\hline \multicolumn{2}{|c|}{ CAS2-homologues } & ${ }^{1}$ MitoProtll & ${ }^{2}$ TargetP \\
\hline 59 & 225 & 0.9848 & $0.876 / \mathrm{RC} 2$ \\
\hline 59 & 224 & 0.9908 & $0.877 / R C 2$ \\
\hline 59 & 254 & 0.9933 & $0.926 / \mathrm{RC} 1$ \\
\hline 75 & 255 & 0.9951 & $0.952 / R C 1$ \\
\hline 58 & 221 & 0.9849 & $0.892 / R C 2$ \\
\hline 71 & 223 & 0.9926 & $0.906 / R C 1$ \\
\hline 46 & 245 & 0.9954 & $0.943 / R C 1$ \\
\hline 44 & 243 & 0.9944 & $0.975 / R C 1$ \\
\hline 33 & 238 & 0.9314 & $0.948 / R C 1$ \\
\hline \begin{tabular}{|l|l|}
37 & \\
\end{tabular} & 239 & 0.9742 & $0.960 / R C 1$ \\
\hline 13 & 293 & 0.7742 & $0.796 / R^{2} 3$ \\
\hline 43 & 225 & 0.9917 & $0.845 / R C 2$ \\
\hline & 1-homologues & & \\
\hline 66 & 216 & 0.9813 & $0.902 / R C 2$ \\
\hline \begin{tabular}{|l|l|l|}
40 & 0 \\
\end{tabular} & 243 & 0.9766 & $0.928 / R C 1$ \\
\hline 39 & 243 & 0.9797 & $0.934 / R C 1$ \\
\hline 61 & 224 & 0.9966 & $0.929 / R C 1$ \\
\hline \begin{tabular}{l|l}
41 \\
\end{tabular} & 227 & 0.9968 & $0.928 / R C 1$ \\
\hline 25 & 262 & 0.9927 & $0.952 / R C 1$ \\
\hline 27 & 260 & 0.9791 & $0.947 / R C 1$ \\
\hline 25 & 255 & 0.9789 & $0.938 / R C 1$ \\
\hline
\end{tabular}

these extra genes were suggestive of horizontal gene transfer from Sordariomycete species (Khaldi and Wolfe 2008). Similarly, the $\alpha$-CA genes in A. oryzae, A. terreus, A. flavus and $A$. niger may originate from such a horizontal gene transfer event (Fig. 5).

Two closely related plant-type $\beta$-CAs have been identified in almost all filamentous ascomycetes. CAS1 and CAS2 homologues show a fairly high degree of sequence identity in all species investigated. This result is suggestive of a gene duplication event of a plant-type $\beta$-CA gene in filamentous ascomycetes (Fig. 5). However, members of the genera Fusarium and Trichoderma (order Hypocreales), exhibit only one of plant-type cas-genes. Within these genera, the casl homologue might have been lost during evolution, or else the gene duplication event resulting in cas 1 and cas 2 took place only in restricted groups of filamentous ascomycetes (Fig. 5). The distribution of plant- and cab-type $\beta$-CA-genes within filamentous ascomycetes suggests that duplication events occurred independently at several times and might reflect specialization events. In eubacteria, specific CA genes in closely related organisms are hypothesized to have been acquired when they were needed for specific physiological reactions (Smith and Ferry 2000). Symbiobacterium thermophilum, a syntrophic bacterium that effectively grows on $\mathrm{CO}_{2}$ generated by other bacteria has lost its CA genes (Nishida et al. 2009). Another example is the Escherichia coli cyanate operon, encoding for a cyanase, a protein of unknown function, and a plant-type $\beta$-CA that generates bicarbonate for the cyanase, which is absent in closely related Salmonella species (Hewett-Emmett and Tashian 1996). Recently, we demonstrated the functionality of the first fungal cyanase in S. macrospora (Elleuche and Pöggeler 2008). In this respect, it would be interesting to find out which of the three fungal $\beta$-CA genes is needed to supply cyanate decomposition with bicarbonate. CAS1 and CAS2 of $S$. macrospora show a high degree of sequence identity to $E$. coli CynT (34.7\% identity in 170 aa overlap; CAS2: $33.9 \%$ in 177 aa; CAS3: $40.0 \%$ in 40 aa) and might be responsible for co-factor supply. 


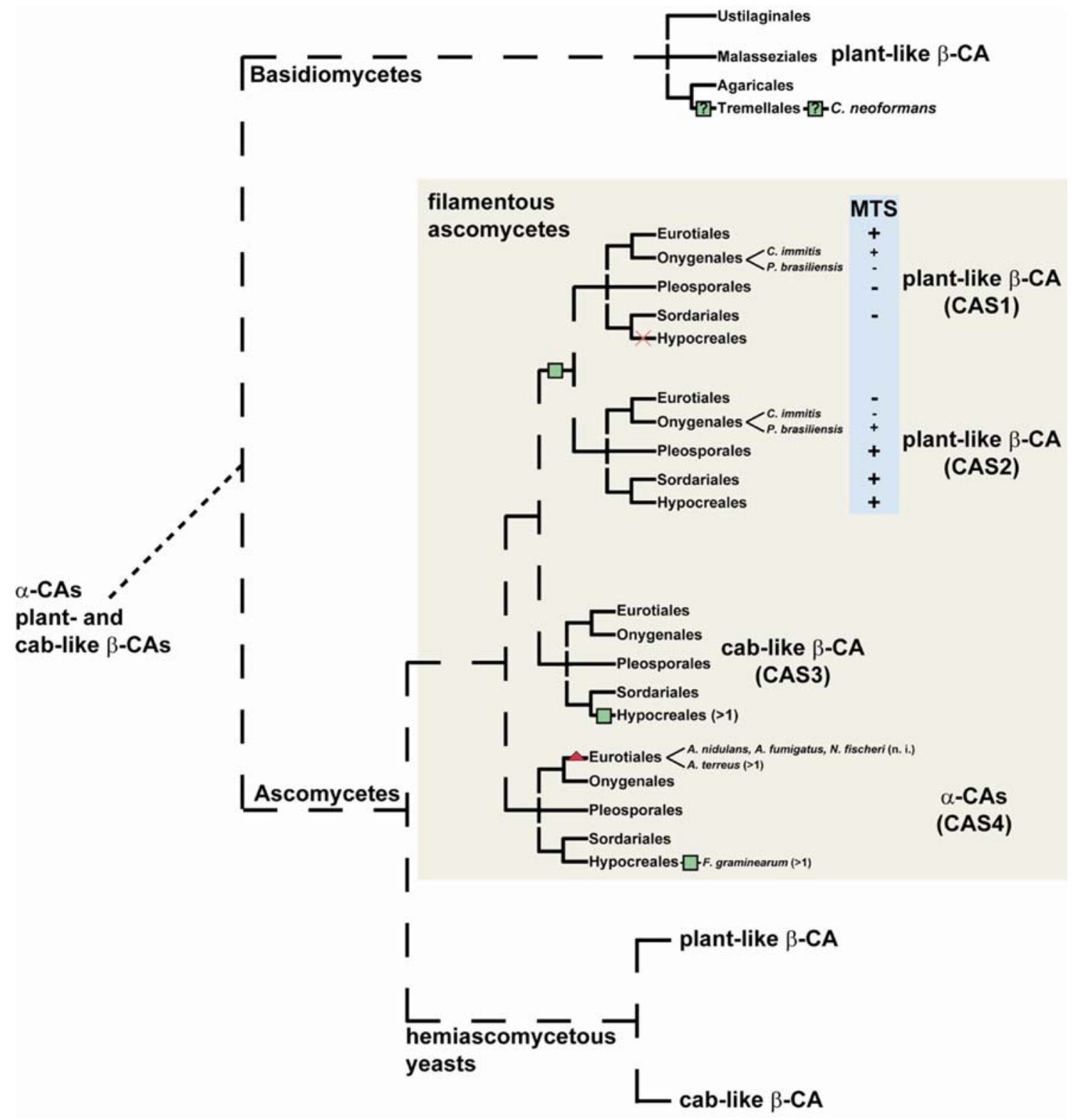

Fig. 5 Schematic illustration of the distribution of CA-genes in fungi. Green boxes indicate gene duplication, red crosses indicate gene loss events and red triangles indicate putative horizontal gene transfers. A question mark in a green box indicates that the gene duplication of the

Furthermore, all fungal $\alpha$ - and $\beta$-CAs used for the generation of the phylogenetic tree revealed no changes in the three essential key amino acids within the catalytic zinccoordinating domain (Fig. 2; Supplementary Figure 1). To our knowledge, no inactive CA-variants have been identified in fungi, as has been described for three inactive cytosolic CARPs in mammals. Loss of CARP enzyme function resulted from substitutions of key amino acids in the catalytic domain (Bellingham et al. 1998; Supuran et al. 2003). $\alpha$-CAs contain three conserved histidines in the catalytic region that function as zinc ligands (Supuran 2008). Other residues that have been shown to be important for
$\beta$-CAs might have occurred either in the order Tremellales or in the genus Cryptococcus. MTS mitochondrial target sequence; n.i. not identified. The tree was drawn according to phylogenetic analysis of Hibbett et al. (2007)

human $\alpha$-CA II enzyme activity are also present in the fungal $\alpha$-CAs (data not shown) (Smith and Ferry 2000). $\beta$-CAs have one conserved histidine and two conserved cysteines coordinating the active zinc site. In addition to these three residues, an aspartic acid and an arginine are also structurally conserved in every known $\beta$-CA sequence (Tripp et al. 2001). These two residues presumably assist in a number of catalytic steps, including substrate binding, proton shuffling and product release, or act as the fourth zinc ligand (Mitsuhashi et al. 2000; Cronk et al. 2001; Smith et al. 2002). These five amino acid residues are completely conserved among the 82 fungal $\beta$-CAs (Supplementary 
Figure 1). However, only a part of the catalytic region was identified in several putative ORFs encoding CAs in the genus Trichoderma. The incomplete CA-ORFs might result from incorrect genome sequences or alternatively, indicate the presence of inactive pseudogenes. These putative CAs (T. atroviride: Triat1/scaffold_3; T. reesei: Trire2/scaffold_5, 1587761-1588374 and 1644451-1644956; T. virens: Trive1/ scaffold_3 and Trive1/scaffold_4) are not considered by the generation of the phylogenetic tree.

Interestingly, blastp and tblastn searches with the cabtype S. macrospora CAS3 identified putative homologues in the pathogenic yeast $C$. albicans (XP_715817) and in Pichia stipitis (XP_001383682.1). This is the first indication of a second cab-type $\beta$-CA-gene in hemiascomycetous yeasts. Determining if the cab-type $\beta$-CA from $C$. albicans is also active and involved in adaptation to varying $\mathrm{CO}_{2}$ conditions, i.e. during host infection as NCE103 (Klengel et al. 2005), would be of great interest. The distribution of cab-type CAs in filamentous ascomycetes is highly diverse. While only one cab-like CA has been identified in the genome from members of the orders Eurotiales, Helotiales, Onygenales, Pleosporales and Sordariales, at least two CAS3-homologues are encoded in species from the genus Fusarium and up to four cab-like CAs have been identified in the genus Trichoderma (Table 1).

In basidiomycetes belonging to the orders Agaricales, Malasseziales and Ustilaginales, we identified only one single plant-type $\beta$-CA in each genome (Table 1). In C. neoformans, the plant-type $\beta$-CAs CAN1 and CAN2 share $36 \%$ identity (Bahn et al. 2005; Schlicker et al. 2009) and cluster together. This suggests that an independent gene duplication event occurred in $C$. neoformans. Since the genome of $C$. neoformans was not duplicated during evolution (Loftus et al. 2005), chromosomal translocation and segmental duplication processes might have resulted in the duplication of the $\beta$-CAs. Only recently has the crystal structure of $C$. neoformans Can2 revealed that the enzyme belongs to the plant-type $\beta$-CAs (Schlicker et al. 2009).

The multiplicity of fungal CA-isoforms leads to the assumption that the proteins are involved in various processes and are targeted to different cellular compartments, as it has been described in plants, algae and animals (Hewett-Emmett and Tashian 1996; Moroney et al. 2001; Supuran 2008; Ynalvez et al. 2008). Because of the high degree of sequence identity, these functional specifications and the subcellular localization events are thought to be quite recent (Hewett-Emmett and Tashian 1996). We suggest that the distribution of putative mitochondrial CAs in fungi of the orders Eurotiales, Sordariales, Pleosporales and Hypocreales might also indicate that the recent acquisition of novel function went along with the translocation of one isoforms into the mitochondria.
In the order Hypocreales, a CAS1-homologue might have gone lost during evolution and one of the multiple cab-type CAs might have taken over the physiological function. Although, we were able to identify a putative MTS in plant-type CAs of most filamentous ascomycetes by bioinformatics means, the functionality of the highly diverse target signals has to be proven experimentally. Recently, we did fluorescence microscopy analyses to investigate the MTS-dependent subcellular localization of S. macrospora CAS2 (S. Elleuche and S. Pöggeler, unpublished data). Similar investigations have to be made with CAS1-homologues of members from the order Eurotiales in order to verify the MTS. The fact that we were not able to locate an alternative start codon in the casl-gene of A. nidulans (XP_663215.1), although the gene structure was annotated in the same manner as in other members, might indicate that the $A$. nidulans sequence is not correct or that the CAS1-homologue is not located to the mitochondria.

Because of their subcellular localization, CAS1-homologues encoded by members of the order Eurotiales, and the CAS2-homologues encoded by members of the orders Sordariales and Hypocreales are probably functional orthologues (Fig. 5). Recently, we investigated the role of the $S$. macrospora cas 2 -gene during fruiting body development and germination of ascospores and showed that the cas 2 gene product is involved in both mechanisms, but the mitochondrial localization is only relevant for the ascospore germination process (S. Elleuche and S. Pöggeler, unpublished data). In the future it would be interesting to illuminate the roles of mitochondrial-localized CAs in other filamentous species.

Fungal $\alpha$ - and $\beta$-class CAs have been annotated in a variety of filamentous and yeast ascomycetes, as well as in some basidiomycetous species. To date, no CAs from the $\gamma$-class or the $\delta$ - and $\zeta$-class have been detected in fungal species. The $\beta$-class encompasses two sub-classes of plantand cab-type representatives. Many filamentous ascomycetes possess one gene encoding a cab-type and two genes encoding plant-type $\beta$-CAs, however some species encode multiple cab-type CAs. This indicates that gene duplication events of ancestors from both types of genes occurred several times during fungal evolution. The characterization of $\beta$-CAs from ancient groups of fungi may shed some light on the origin of this class of genes.

In filamentous ascomycetes, plant-type $\beta$-CAs include cytosolic and mitochondrial members, whereas cab-type $\beta$-CAs seem to be exclusively cytoplasmic enzymes. Interestingly, within the orders Eurotiales and Sordariales, non-homologues $\beta$-CAs are targeted to the mitochondria. Therefore, we assume that the MTS-dependent protein transport of fungal $\beta$-CAs may have evolved in parallel with the evolution of new, mitochondrial-specific functions. 
Acknowledgments The authors thank Yasmine Bernhards for critically reading the manuscript. English language editing of the manuscript was performed by International Science Editing, Ireland.

Open Access This article is distributed under the terms of the Creative Commons Attribution Noncommercial License which permits any noncommercial use, distribution, and reproduction in any medium, provided the original author(s) and source are credited.

\section{References}

Alber BE, Ferry JG (1994) A carbonic anhydrase from the archaeon Methanosarcina thermophila. Proc Natl Acad Sci USA 91:69096913

Altschul SF et al (1997) Gapped BLAST and PSI-BLAST: a new generation of protein database search programs. Nucleic Acids Res 25:3389-3402

Amoroso G, Morell-Avrahov L, Muller D, Klug K, Sültemeyer D (2005) The gene NCE103 (YNL036w) from Saccharomyces cerevisiae encodes a functional carbonic anhydrase and its transcription is regulated by the concentration of inorganic carbon in the medium. Mol Microbiol 56:549-558

Bahn YS, Mühlschlegel FA (2006) $\mathrm{CO}_{2}$ sensing in fungi and beyond. Curr Opin Microbiol 9:572-578

Bahn YS, Cox GM, Perfect JR, Heitman J (2005) Carbonic anhydrase and $\mathrm{CO}_{2}$ sensing during Cryptococcus neoformans growth, differentiation, and virulence. Curr Biol 15:2013-2020

Bellingham J, Gregory-Evans K, Gregory-Evans CY (1998) Sequence and tissue expression of a novel human carbonic anhydrase-related protein, CARP-2, mapping to chromosome 19q13.3. Biochem Biophys Res Commun 253:364-367

Claros MG, Vincens P (1996) Computational method to predict mitochondrially imported proteins and their targeting sequences. Eur J Biochem 241:779-786

Cronk JD, Endrizzi JA, Cronk MR, O’Neill JW, Zhang KY (2001) Crystal structure of E. coli beta-carbonic anhydrase, an enzyme with an unusual pH-dependent activity. Protein Sci 10:911-922

Elleuche S, Pöggeler S (2008) A cyanase is transcriptionally regulated by arginine and involved in cyanate decomposition in Sordaria macrospora. Fungal Genet Biol 45:1458-1469

Emanuelsson O, Brunak S, von Heijne G, Nielsen H (2007) Locating proteins in the cell using TargetP, SignalP and related tools. Nat Protoc 2:953-971

Espagne E et al (2008) The genome sequence of the model ascomycete fungus Podospora anserina. Genome Biol 9:R77

Götz R, Gnann A, Zimmermann FK (1999) Deletion of the carbonic anhydrase-like gene NCE103 of the yeast Saccharomyces cerevisiae causes an oxygen-sensitive growth defect. Yeast 15:855-864

Hewett-Emmett D, Tashian RE (1996) Functional diversity, conservation, and convergence in the evolution of the alpha-, beta-, and gamma-carbonic anhydrase gene families. Mol Phylogenet Evol 5:50-77

Hibbett DS et al (2007) A higher-level phylogenetic classification of the Fungi. Mycol Res 111:509-547

Khaldi N, Wolfe KH (2008) Elusive origins of the extra genes in Aspergillus oryzae. PLoS One 3:e3036

Kimber MS, Pai EF (2000) The active site architecture of Pisum sativum beta-carbonic anhydrase is a mirror image of that of alphacarbonic anhydrases. EMBO J 19:1407-1418

Kivelä AJ, Kivelä J, Saarnio J, Parkkila S (2005) Carbonic anhydrases in normal gastrointestinal tract and gastrointestinal tumours. World J Gastroenterol 11:155-163
Klengel T et al (2005) Fungal adenylyl cyclase integrates $\mathrm{CO}_{2}$ sensing with cAMP signaling and virulence. Curr Biol 15:2021-2026

Lane TW, Saito MA, George GN, Pickering IJ, Prince RC, Morel FM (2005) Biochemistry: a cadmium enzyme from a marine diatom. Nature 435:42

Liljas A, Laurberg M (2000) A wheel invented three times. The molecular structures of the three carbonic anhydrases. EMBO Rep $1: 16-17$

Loftus BJ et al (2005) The genome of the basidiomycetous yeast and human pathogen Cryptococcus neoformans. Science 307:13211324

McGuffin LJ, Bryson K, Jones DT (2000) The PSIPRED protein structure prediction server. Bioinformatics 16:404-405

Mitsuhashi S et al (2000) X-ray structure of beta-carbonic anhydrase from the red alga, Porphyridium purpureum, reveals a novel catalytic site for $\mathrm{CO}_{2}$ hydration. J Biol Chem 275:5521-5526

Mogensen EG et al (2006) Cryptococcus neoformans senses $\mathrm{CO}_{2}$ through the carbonic anhydrase Can 2 and the adenylyl cyclase Cac1. Eukaryot Cell 5:103-111

Mori K et al (1999) Isolation and characterization of CA XIV, a novel membrane-bound carbonic anhydrase from mouse kidney. J Biol Chem 274:15701-15705

Moroney JV, Bartlett SG, Samuelsson G (2001) Carbonic anhydrases in plants and algae. Plant Cell Environ 24:141-153

Nishida H, Beppu T, Ueda K (2009) Symbiobacterium lost carbonic anhydrase in the course of evolution. J Mol Evol 68:90-96

Page RD (1996) TreeView: an application to display phylogenetic trees on personal computers. Comput Appl Biosci 12:357-358

Park H, Song B, Morel FM (2007) Diversity of the cadmium-containing carbonic anhydrase in marine diatoms and natural waters. Environ Microbiol 9:403-413

Sawaya MR et al (2006) The structure of beta-carbonic anhydrase from the carboxysomal shell reveals a distinct subclass with one active site for the price of two. J Biol Chem 281:7546-7555

Schlicker C et al (2009) Structure and inhibition of the $\mathrm{CO}_{2}$-sensing carbonic anhydrase Can 2 from the pathogenic fungus Cryptococcus neoformans. J Mol Biol 385:1207-1220

Smith KS, Ferry JG (1999) A plant-type (beta-class) carbonic anhydrase in the thermophilic methanoarchaeon Methanobacterium thermoautotrophicum. J Bacteriol 181:6247-6253

Smith KS, Ferry JG (2000) Prokaryotic carbonic anhydrases. FEMS Microbiol Rev 24:335-366

Smith KS, Ingram-Smith C, Ferry JG (2002) Roles of the conserved aspartate and arginine in the catalytic mechanism of an archaeal beta-class carbonic anhydrase. J Bacteriol 184:4240-4245

So AK, Espie GS, Williams EB, Shively JM, Heinhorst S, Cannon GC (2004) A novel evolutionary lineage of carbonic anhydrase (epsilon class) is a component of the carboxysome shell. J Bacteriol 186:623-630

Supuran CT (2008) Carbonic anhydrases-an overview. Curr Pharm Des 14:603-614

Supuran CT, Scozzafava A, Casini A (2003) Carbonic anhydrase inhibitors. Med Res Rev 23:146-189

Thompson JD, Gibson TJ, Higgins DG (2002) Multiple sequence alignment using ClustalW and ClustalX. In: Current protocols in bioinformatics. Wiley \& Sons, New York (Chapter 2, Unit 2.3)

Tripp BC, Smith K, Ferry JG (2001) Carbonic anhydrase: new insights for an ancient enzyme. J Biol Chem 276:48615-48618

Ynalvez RA, Xiao Y, Ward AS, Cunnusamy K, Moroney JV (2008) Identification and characterization of two closely related beta-carbonic anhydrases from Chlamydomonas reinhardtii. Physiol Plant 133:15-26 